\title{
Permanence and global attractivity in a discrete Lotka-Volterra predator-prey model with delays
}

\author{
Changjin $\mathrm{Xu}^{1 *}$, Yusen $\mathrm{Wu}^{2}$ and Lin $\mathrm{Lu}^{1}$
}

"Correspondence: xcj403@126.com ${ }^{1}$ Guizhou Key Laboratory of Economics System Simulation, School of Mathematics and Statistics, Guizhou University of Finance and Economics, Guiyang, 550004, P.R. China

Full list of author information is available at the end of the article

\section{空 Springer}

\begin{abstract}
In this paper, we deal with a discrete Lotka-Volterra predator-prey model with time-varying delays. For the general non-autonomous case, sufficient conditions which ensure the permanence and global stability of the system are obtained by using differential inequality theory. For the periodic case, sufficient conditions which guarantee the existence of a unique globally stable positive periodic solution are established. The paper ends with some interesting numerical simulations that illustrate our analytical predictions.

MSC: 34K20; 34C25; 92D25

Keywords: Lotka-Volterra predator-prey model; permanence; global attractivity; delay
\end{abstract}

\section{Introduction}

After the pioneering work of Berryman [1] in 1992, the dynamic relationship between predators and their preys has become one of the dominant themes in both ecology and mathematical ecology due to its universal existence and importance. Dynamic nature (including the local and global stability of the equilibrium, the persistence, permanence and extinction of species, the existence of periodic solutions and positive almost periodic solutions, bifurcation and chaos and so on) of predator-prey models has been investigated in a number of notable studies [2-26]. In many applications, the nature of permanence is of great interest. For example, Fan and Li [27] made a theoretical discussion on the permanence of a delayed ratio-dependent predator-prey model with Holling-type functional response. Chen [28] addressed the permanence of a discrete $n$-species delayed foodchain system. Zhao and Jiang [29] focused on the permanence and extinction for a nonautonomous Lotka-Volterra system. Chen [30] analyzed the permanence and global attractivity of a Lotka-Volterra competition system with feedback control. Zhao and Teng et al. [31] established the permanence criteria for delayed discrete non-autonomous-species Kolmogorov systems. For more research on the permanence behavior of predator-prey models, one can see [32-44].

In 2010, Lv et al. [45] investigated the existence and global attractivity of a periodic solution to the following Lotka-Volterra predator-prey system:

○2014 Xu et al.; licensee Springer. This is an Open Access article distributed under the terms of the Creative Commons Attribution License (http://creativecommons.org/licenses/by/2.0), which permits unrestricted use, distribution, and reproduction in any medium, provided the original work is properly cited. 


$$
\left\{\begin{aligned}
\frac{d x_{1}(t)}{d t}= & x_{1}(t)\left[r_{1}(t)-a_{11}(t) x_{1}\left(t-\tau_{11}(t)\right)-a_{12}(t) x_{2}\left(t-\tau_{12}(t)\right)\right. \\
& \left.-a_{13}(t) x_{3}\left(t-\tau_{13}(t)\right)\right] \\
\frac{d x_{2}(t)}{d t}= & x_{2}(t)\left[-r_{2}(t)+a_{21}(t) x_{1}\left(t-\tau_{21}(t)\right)-a_{22}(t) x_{2}\left(t-\tau_{22}(t)\right)\right. \\
& \left.-a_{23}(t) x_{3}\left(t-\tau_{23}(t)\right)\right] \\
\frac{d x_{3}(t)}{d t}= & x_{3}(t)\left[-r_{3}(t)+a_{31}(t) x_{1}\left(t-\tau_{31}(t)\right)-a_{32}(t) x_{2}\left(t-\tau_{32}(t)\right)\right. \\
& \left.-a_{33}(t) x_{3}\left(t-\tau_{33}(t)\right)\right]
\end{aligned}\right.
$$

where $x_{1}(t)$ denotes the density of prey species at time $t, x_{2}(t)$ and $x_{3}(t)$ stand for the density of predator species at time $t, r_{i}, a_{i j} \in C(R,[0, \infty))$ and $\tau_{i j}(t)>0(i, j=1,2,3)$. Using Krasnoselskii's fixed point theorem and constructing the Lyapunov function, Lv et al. obtained a set of easily verifiable sufficient conditions which guarantee the permanence and global attractivity of system (1.1).

Many authors have argued that discrete time models governed by difference equations are more appropriate to describe the dynamics relationship among populations than continuous ones when the populations have non-overlapping generations. Moreover, discrete time models can also provide efficient models of continuous ones for numerical simulations $[4,16,46]$. Thus it is reasonable and interesting to investigate discrete time systems governed by difference equations. The principal object of this article is to propose a discrete analogue system (1.1) and explore its dynamics.

Following the ideas of $[4,11]$, we will discretize system (1.1). Assume that the average growth rates in system (1.1) change at regular intervals of time, then we can obtain the following modified system:

$$
\left\{\begin{aligned}
\frac{\dot{x}_{1}(t)}{x_{1}(t)}= & r_{1}([t])-a_{11}([t]) x_{1}\left([t]-\tau_{11}([t])\right)-a_{12}([t]) x_{2}\left([t]-\tau_{12}([t])\right) \\
& -a_{13}([t]) x_{3}\left([t]-\tau_{13}([t])\right) \\
\frac{\dot{x}_{2}(t)}{x_{2}(t)}= & -r_{2}([t])+a_{21}([t]) x_{1}\left([t]-\tau_{21}([t])\right)-a_{22}([t]) x_{2}\left([t]-\tau_{22}([t])\right) \\
& -a_{23}([t]) x_{3}\left([t]-\tau_{23}([t])\right) \\
\frac{\dot{x}_{3}(t)}{x_{3}(t)}= & -r_{3}([t])+a_{31}([t]) x_{1}\left([t]-\tau_{31}([t])\right)-a_{32}([t]) x_{2}\left([t]-\tau_{32}([t])\right) \\
& -a_{33}([t]) x_{3}\left([t]-\tau_{33}([t])\right)
\end{aligned}\right.
$$

where $[t]$ denotes the integer part of $t, t \in(0,+\infty)$ and $t \neq 0,1,2, \ldots$ We integrate (1.2) on any interval of the form $[k, k+1), k=0,1,2, \ldots$, and obtain

$$
\left\{\begin{aligned}
x_{1}(t)= & x_{1}(k) \exp \left\{\left[r_{1}(k)-a_{11}(k) x_{1}\left(k-\tau_{11}(k)\right)-a_{12}(k) x_{2}\left(k-\tau_{12}(k)\right)\right.\right. \\
& \left.\left.-a_{13}(k) x_{3}\left(k-\tau_{13}(k)\right)\right](t-k)\right\} \\
x_{2}(t)= & x_{2}(k) \exp \left\{\left[-r_{2}(k)+a_{21}(k) x_{1}\left(k-\tau_{21}(k)\right)-a_{22}(k) x_{2}\left(k-\tau_{22}(k)\right)\right.\right. \\
& \left.\left.-a_{23}(k) x_{3}\left(k-\tau_{23}(k)\right)\right](t-k)\right\} \\
x_{3}(t)= & x_{3}(k) \exp \left\{\left[-r_{3}(k)+a_{31}(k) x_{1}\left(k-\tau_{31}(k)\right)-a_{32}(k) x_{2}\left(k-\tau_{32}(k)\right)\right.\right. \\
& \left.\left.-a_{33}(k) x_{3}\left(k-\tau_{33}(k)\right)\right](t-k)\right\}
\end{aligned}\right.
$$

where for $k \leq t<k+1, k=0,1,2, \ldots$. 
Let $t \rightarrow k+1$, then (1.3) takes the following form:

$$
\left\{\begin{aligned}
x_{1}(k+1)= & x_{1}(k) \exp \left\{r_{1}(k)-a_{11}(k) x_{1}\left(k-\tau_{11}(k)\right)-a_{12}(k) x_{2}\left(k-\tau_{12}(k)\right)\right. \\
& \left.-a_{13}(k) x_{3}\left(k-\tau_{13}(k)\right)\right\} \\
x_{2}(k+1)= & x_{2}(k) \exp \left\{-r_{2}(k)+a_{21}(k) x_{1}\left(k-\tau_{21}(k)\right)-a_{22}(k) x_{2}\left(k-\tau_{22}(k)\right)\right. \\
& \left.-a_{23}(k) x_{3}\left(k-\tau_{23}(k)\right)\right\} \\
x_{3}(k+1)= & x_{3}(k) \exp \left\{-r_{3}(k)+a_{31}(k) x_{1}\left(k-\tau_{31}(k)\right)-a_{32}(k) x_{2}\left(k-\tau_{32}(k)\right)\right. \\
& \left.-a_{33}(k) x_{3}\left(k-\tau_{33}(k)\right)\right\}
\end{aligned}\right.
$$

which is a discrete time analogue of system (1.1), where $k=0,1,2, \ldots$.

For the point of view of biology, we shall consider (1.4) together with the initial conditions $x_{i}(0) \geq 0(i=1,2,3)$. The principal object of this article is to explore the dynamics of system (1.4) applying the differential inequality theory to study the permanence of system (1.4). Using the method of Lyapunov function, we investigate the global asymptotic stability of system (1.4).

We assume that the coefficients of system (1.4) satisfy the following:

(H1) $r_{i}, a_{i j}, \tau_{i j}$ with $i, j=1,2,3$ are non-negative sequences bounded above and below by positive constants.

Let $\tau=\sup _{1 \leq i, j \leq 3, k \in Z}\left\{\tau_{i j}(k)\right\}$. We consider (1.4) together with the following initial conditions:

$$
x_{i}(\theta)=\varphi_{i}(\theta) \geq 0, \quad \theta \in N[-\tau, 0]=\{-\tau,-\tau+1, \ldots, 0\}, \varphi_{i}(0)>0
$$

It is not difficult to see that the solutions of (1.4) and (1.5) are well defined for all $k \geq 0$ and satisfy

$$
x_{i}(k)>0 \quad \text { for } k \in Z, i=1,2,3 .
$$

The remainder of the paper is organized as follows. In Section 2, basic definitions and lemmas are given, some sufficient conditions for the permanence of system (1.4) are established. In Section 3, a series of sufficient conditions for the global stability of system (1.4) are included. The existence and stability of system (1.4) are analyzed in Section 4. In Section 5 , we give an example which shows the feasibility of the main results. Conclusions are presented in Section 6.

\section{Permanence}

For convenience, in the following discussion, we always use the notations:

$$
f^{l}=\inf _{k \in Z} f(k), \quad f^{u}=\sup _{k \in Z} f(k)
$$

where $f(k)$ is a non-negative sequence bounded above and below by positive constants. In order to obtain the main result of this paper, we shall first state the definition of permanence and several lemmas which will be useful in the proof of the main result.

Definition 2.1 [47] We say that system (1.4) is permanent if there are positive constants $M$ and $m$ such that each positive solution $\left(x_{1}(k), x_{2}(k), x_{3}(k)\right)$ of system (1.4) satisfies

$$
m \leq \lim _{k \rightarrow+\infty} \inf x_{i}(k) \leq \lim _{k \rightarrow+\infty} \sup x_{i}(k) \leq M \quad(i=1,2,3) .
$$


Lemma 2.1 [47] Assume that $\{x(k)\}$ satisfies $x(k)>0$ and

$$
x(k+1) \leq x(k) \exp \{a(k)-b(k) x(k)\}
$$

for $k \in N$, where $a(k)$ and $b(k)$ are non-negative sequences bounded above and below by positive constants. Then

$$
\lim _{k \rightarrow+\infty} \sup x(k) \leq \frac{1}{b^{l}} \exp \left(a^{u}-1\right)
$$

Lemma 2.2 [47] Assume that $\{x(k)\}$ satisfies

$$
x(k+1) \geq x(k) \exp \{a(k)-b(k) x(k)\}, \quad k \geq N_{0},
$$

$\lim _{k \rightarrow+\infty} \sup x(k) \leq x^{*}$ and $x\left(N_{0}\right)>0$, where $a(k)$ and $b(k)$ are non-negative sequences bounded above and below by positive constants and $N_{0} \in N$. Then

$$
\lim _{k \rightarrow+\infty} \inf x(k) \geq \min \left\{\frac{a^{l}}{b^{u}} \exp \left\{a^{l}-b^{u} x^{*}\right\}, \frac{a^{l}}{b^{u}}\right\} .
$$

Now we state our permanence result for system (1.4).

Theorem 2.1 Let $M_{1}, M_{2}, M_{3}$ and $m_{1}$ be defined by (2.4), (2.10), (2.15) and (2.20), respectively. In addition to condition (H1), assume that the following conditions:

$$
a_{21}^{u} M_{1}>r_{2}^{l}, \quad a_{22}^{u} M_{1}>r_{2}^{l}, \quad a_{31}^{u} M_{1}>r_{3}^{l}
$$

and

$$
\text { (H3) } \quad r_{1}^{l}>a_{12}^{u} M_{1}+a_{13}^{u} M_{3}, \quad r_{2}^{u}>a_{23}^{u} M_{3}, \quad a_{31}^{l} m_{1}>r_{3}^{u}+a_{32}^{u} M_{2}
$$

hold, then system (1.4) is permanent, that is, there exist positive constants $m_{i}, M_{i}(i=1,2,3)$ which are independent of the solution of system (1.4), such that for any positive solution $\left(x_{1}(k), x_{2}(k), x_{3}(k)\right)$ of system (1.4) with the initial condition $x_{i}(0) \geq 0(i=1,2,3)$, one has

$$
m_{i} \leq \lim _{k \rightarrow+\infty} \inf x_{i}(k) \leq \lim _{k \rightarrow+\infty} \sup x_{i}(k) \leq M_{i}
$$

Proof Let $\left(x_{1}(k), x_{2}(k), x_{3}(k)\right)$ be any positive solution of system (1.4) with the initial condition $\left(x_{1}(0), x_{2}(0), x_{3}(0)\right)$. It follows from the first equation of system (1.4) that

$$
\begin{aligned}
x(k+1)= & x_{1}(k) \exp \left\{\left[r_{1}(k)-a_{11}(k) x_{1}\left(k-\tau_{11}(k)\right)\right.\right. \\
& \left.\left.-a_{12}(k) x_{2}\left(k-\tau_{12}(k)\right)-a_{13}(k) x_{3}\left(k-\tau_{13}(k)\right)\right]\right\} \\
\leq & x_{1}(k) \exp \left\{r_{1}(k)\right\} \leq x_{1}(k) \exp \left\{r_{1}^{u}\right\} .
\end{aligned}
$$

It follows from (2.1) that

$$
x_{1}\left(k-\tau_{11}(k)\right) \geq x_{1}(k) \exp \left\{-r_{1}^{u} \tau^{u}\right\} .
$$


Substituting (2.2) into the first equation of system (1.4), we get

$$
x_{1}(k+1) \leq x_{1}(k)\left[r_{1}^{u}-a_{11}^{l} \exp \left\{-r_{1}^{u} \tau^{u}\right\} x_{1}(k)\right]
$$

It follows from (2.3) and Lemma 2.1 that

$$
\lim _{k \rightarrow+\infty} \sup x_{1}(k) \leq \frac{1}{a_{11}^{l}} \exp \left\{r_{1}^{u} \tau^{u}+r_{1}^{u}-1\right\}:=M_{1} .
$$

For any positive constant $\varepsilon>0$, it follows from (2.4) that there exists $N_{1}>0$ such that for all $k>N_{1}$,

$$
x_{1}(k) \leq M_{1}+\varepsilon \text {. }
$$

For $k \geq N_{1}+\tau^{u}$, from (2.5) and the second equation of system (1.4), we have

$$
\begin{aligned}
x_{2}(k+1)= & x_{2}(k) \exp \left\{\left[-r_{2}(k)+a_{21}(k) x_{1}\left(k-\tau_{21}(k)\right)\right.\right. \\
& \left.\left.-a_{22}(k) x_{2}\left(k-\tau_{22}(k)\right)-a_{23}(k) x_{3}\left(k-\tau_{23}(k)\right)\right]\right\} \\
\leq & x_{2}(k) \exp \left\{\left[-r_{2}(k)+a_{21}(k) x_{1}\left(k-\tau_{21}(k)\right)\right]\right\} \\
\leq & x_{1}(k) \exp \left\{\left[-r_{2}^{l}+a_{21}^{u}\left(M_{1}+\varepsilon\right)\right]\right\},
\end{aligned}
$$

which leads to

$$
x_{2}\left(k-\tau_{22}(k)\right) \geq x_{2}(k) \exp \left\{\left[r_{2}^{l}-a_{21}^{u}\left(M_{1}+\varepsilon\right)\right] \tau^{u}\right\} .
$$

Substituting (2.7) into the second equation of system (1.4), we have

$$
\begin{aligned}
x_{2}(k+1)= & x_{2}(k) \exp \left\{\left[-r_{2}(k)+a_{21}(k) x_{1}\left(k-\tau_{21}(k)\right)\right.\right. \\
& \left.\left.-a_{22}(k) x_{2}\left(k-\tau_{22}(k)\right)-a_{23}(k) x_{3}\left(k-\tau_{23}(k)\right)\right]\right\} \\
\leq & x_{2}(k) \exp \left\{\left[-r_{2}(k)+a_{21}(k) x_{1}\left(k-\tau_{21}(k)\right)\right.\right. \\
& \left.\left.-a_{22}(k) x_{2}\left(k-\tau_{22}(k)\right)\right]\right\} \\
\leq & x_{2}(k)\left[-r_{2}^{l}+a_{21}^{u}\left(M_{1}+\varepsilon\right)-a_{22}^{l} \exp \left\{\left[r_{2}^{l}-a_{21}^{u}\left(M_{1}+\varepsilon\right)\right] \tau^{u}\right\} x_{2}(k)\right] .
\end{aligned}
$$

Thus it follows from Lemma 2.1 and (2.8) that

$$
\lim _{k \rightarrow+\infty} \sup x_{2}(k) \leq \frac{\exp \left\{-r_{2}^{l}+a_{22}^{u}\left(M_{1}+\varepsilon\right)-1\right\}}{a_{22}^{l} \exp \left\{\left[r_{2}^{l}-a_{21}^{u}\left(M_{1}+\varepsilon\right)\right] \tau^{u}\right\}}
$$

Setting $\varepsilon \rightarrow 0$, we obtain

$$
\lim _{k \rightarrow+\infty} \sup x_{2}(k) \leq \frac{\exp \left\{-r_{2}^{l}+a_{22}^{u} M_{1}\right\}}{a_{22}^{l} \exp \left\{\left(r_{2}^{l}-a_{21}^{u} M_{1}\right) \tau^{u}\right\}}:=M_{2} \text {. }
$$


For $k \geq N_{1}+\tau^{u}$, from (2.5) and the third equation of system (1.4), we have

$$
\begin{aligned}
x_{3}(k+1)= & x_{3}(k)\left[-r_{3}(k)+a_{31}(k) x_{1}\left(k-\tau_{31}(k)\right)\right. \\
& \left.-a_{32}(k) x_{2}\left(k-\tau_{32}(k)\right)-a_{33}(k) x_{3}\left(k-\tau_{33}(k)\right)\right] \\
\leq & x_{3}(k)\left[-r_{3}(k)+a_{31}(k) x_{1}\left(k-\tau_{31}(k)\right)\right] \leq x_{3}(k)\left[-r_{3}^{l}+a_{31}^{u}\left(M_{1}+\varepsilon\right)\right],
\end{aligned}
$$

which leads to

$$
x_{3}\left(k-\tau_{33}(k)\right) \geq x_{3}(k) \exp \left\{\left[r_{3}^{l}-a_{31}^{u}\left(M_{1}+\varepsilon\right)\right] \tau^{u}\right\} .
$$

Substituting (2.12) into the third equation of system (1.4) leads to

$$
x_{3}(k+1) \leq x_{3}(k)\left\{-r_{3}^{l}+a_{31}^{u}\left(M_{1}+\varepsilon\right)-a_{33}^{l} \exp \left\{\left[r_{3}^{l}-a_{31}^{u}\left(M_{1}+\varepsilon\right)\right] \tau^{u}\right\} x_{3}(k)\right\} .
$$

In view of Lemma 2.1 and (2.13), one has

$$
\lim _{k \rightarrow+\infty} \sup x_{3}(k) \leq \frac{\exp \left\{-r_{3}^{l}+a_{31}^{u}\left(M_{1}+\varepsilon\right)-1\right\}}{a_{33}^{l} \exp \left\{\left[r_{3}^{l}-a_{31}^{u}\left(M_{1}+\varepsilon\right)\right] \tau^{u}\right\}}
$$

Setting $\varepsilon \rightarrow 0$, we get

$$
\lim _{k \rightarrow+\infty} \sup x_{3}(k) \leq \frac{-r_{3}^{l}+a_{31}^{u} M_{1}}{a_{33}^{l} \exp \left\{\left(r_{3}^{l}-a_{31}^{u} M_{1}\right) \tau^{u}\right\}}:=M_{3} .
$$

For $k \geq N_{1}+\tau^{u}$, it follows from the first equation of system (1.4) that

$$
\begin{aligned}
x_{1}(k+1)= & x_{1}(k)\left[r_{1}(k)-a_{11}(k) x_{1}\left(k-\tau_{11}(k)\right)\right. \\
& \left.-a_{12}(k) x_{2}\left(k-\tau_{12}(k)\right)-a_{13}(k) x_{3}\left(k-\tau_{13}(k)\right)\right] \\
\geq & x_{1}(k)\left[r_{1}^{l}-a_{11}^{u}\left(M_{1}+\varepsilon\right)-a_{12}^{u}\left(M_{2}+\varepsilon\right)-a_{13}^{u}\left(M_{3}+\varepsilon\right)\right],
\end{aligned}
$$

which leads to

$$
x_{1}\left(k-\tau_{11}(k)\right) \leq x_{1}(k) \exp \left\{-\left[r_{1}^{l}-a_{11}^{u}\left(M_{1}+\varepsilon\right)-a_{12}^{u}\left(M_{2}+\varepsilon\right)-a_{13}^{u}\left(M_{3}+\varepsilon\right)\right] \tau^{u}\right\}
$$

Substituting (2.17) into the first equation of system (1.4), we obtain

$$
\begin{aligned}
x_{1}(k+1) \geq & x_{1}(k)\left\{r_{1}^{l}-a_{12}^{u}\left(M_{2}+\varepsilon\right)-a_{13}^{u}\left(M_{3}+\varepsilon\right)\right. \\
& -a_{11}^{u} \exp \left\{-\left[r_{1}^{l}-a_{11}^{u}\left(M_{1}+\varepsilon\right)-a_{12}^{u}\left(M_{2}+\varepsilon\right)\right.\right. \\
& \left.\left.\left.-a_{13}^{u}\left(M_{3}+\varepsilon\right)\right] \tau^{u}\right\} x_{1}(k)\right\} .
\end{aligned}
$$

According to Lemma 2.2, it follows from (2.18) that

$$
\lim _{k \rightarrow+\infty} \inf x_{1}(k) \geq \min \left\{A_{1 \varepsilon}, A_{2 \varepsilon}\right\}
$$


where

$$
\begin{aligned}
A_{1 \varepsilon}= & \frac{r_{1}^{l}-a_{12}^{u}\left(M_{2}+\varepsilon\right)-a_{13}^{u}\left(M_{3}+\varepsilon\right)}{a_{11}^{u} \exp \left\{-\left[r_{1}^{l}-a_{11}^{u}\left(M_{1}+\varepsilon\right)-a_{12}^{u}\left(M_{2}+\varepsilon\right)-a_{13}^{u}\left(M_{3}+\varepsilon\right)\right] \tau^{u}\right\}} \\
& \times \exp \left\{r_{1}^{l}-a_{12}^{u}\left(M_{2}+\varepsilon\right)-a_{13}^{u}\left(M_{3}+\varepsilon\right)-a_{11}^{u} \exp \left\{-\left[r_{1}^{l}-a_{11}^{u}\left(M_{1}+\varepsilon\right)\right.\right.\right. \\
& \left.\left.\left.-a_{12}^{u}\left(M_{2}+\varepsilon\right)-a_{13}^{u}\left(M_{3}+\varepsilon\right)\right] \tau^{u}\right\}\right\} M_{1}, \\
A_{2 \varepsilon}= & \frac{r_{1}^{l}-a_{12}^{u}\left(M_{2}+\varepsilon\right)-a_{13}^{u}\left(M_{3}+\varepsilon\right)}{a_{11}^{u} \exp \left\{-\left[r_{1}^{l}-a_{11}^{u}\left(M_{1}+\varepsilon\right)-a_{12}^{u}\left(M_{2}+\varepsilon\right)-a_{13}^{u}\left(M_{3}+\varepsilon\right)\right] \tau^{u}\right\}} .
\end{aligned}
$$

Setting $\varepsilon \rightarrow 0$ in (2.19), we can get

$$
\lim _{k \rightarrow+\infty} \inf x_{1}(k) \geq \frac{1}{2} \min \left\{A_{1}, A_{2}\right\}:=m_{1}
$$

where

$$
\begin{aligned}
A_{1}= & \frac{r_{1}^{l}-a_{12}^{u} M_{2}-a_{13}^{u} M_{3}}{a_{11}^{u} \exp \left\{-\left[r_{1}^{l}-a_{11}^{u} M_{1}-a_{12}^{u} M_{2}-a_{13}^{u} M_{3}\right] \tau^{u}\right\}} \\
& \times \exp \left\{r_{1}^{l}-a_{12}^{u} M_{2}-a_{13}^{u} M_{3}-a_{11}^{u} \exp \left\{-\left[r_{1}^{l}-a_{11}^{u} M_{1}\right.\right.\right. \\
& \left.\left.\left.-a_{12}^{u} M_{2}-a_{13}^{u} M_{3}\right] \tau^{u}\right\}\right\} M_{1}, \\
A_{2}= & \frac{r_{1}^{l}-a_{12}^{u} M_{2}-a_{13}^{u} M_{3}}{a_{11}^{u} \exp \left\{-\left[r_{1}^{l}-a_{11}^{u} M_{1}-a_{12}^{u} M_{2}-a_{13}^{u} M_{3}\right] \tau^{u}\right\}} .
\end{aligned}
$$

For $k \geq N_{1}+\tau^{u}$, from the second equation of system (1.4), we have

$$
\begin{aligned}
x_{2}(k+1)= & x_{2}(k)\left[-r_{2}(k)+a_{21}(k) x_{1}\left(k-\tau_{21}(k)\right)\right. \\
& \left.-a_{22}(k) x_{2}\left(k-\tau_{22}(k)\right)-a_{23}(k) x_{3}\left(k-\tau_{23}(k)\right)\right] \\
\geq & x_{2}(t)\left[-r_{2}^{u}+a_{21}^{l}\left(m_{1}-\varepsilon\right)-a_{22}^{u}\left(M_{2}+\varepsilon\right)-a_{23}^{u}\left(M_{3}+\varepsilon\right)\right],
\end{aligned}
$$

which leads to

$$
x_{2}\left(k-\tau_{22}(k)\right) \leq x_{2}(k) \exp \left\{\left[r_{2}^{u}-a_{21}^{l}\left(m_{1}-\varepsilon\right)+a_{22}^{u}\left(M_{2}+\varepsilon\right)+a_{23}^{u}\left(M_{3}+\varepsilon\right)\right] \tau^{u}\right\} .
$$

Substituting (2.22) into the second equation of system (1.4) leads to

$$
\begin{aligned}
x_{2}(k+1) \geq & x_{2}(k)\left\{r_{2}^{u}-a_{22}^{u} \exp \left\{\left[r_{2}^{u}-a_{21}^{l}\left(m_{1}-\varepsilon\right)+a_{22}^{u}\left(M_{2}+\varepsilon\right)+a_{23}^{u}\left(M_{3}+\varepsilon\right)\right] \tau^{u}\right\} x_{2}(k)\right. \\
& \left.-a_{23}^{u}\left(M_{3}+\varepsilon\right)\right\} .
\end{aligned}
$$

By Lemma 2.2 and (2.23), we can get

$$
\lim _{k \rightarrow+\infty} \inf x_{2}(k) \geq \min \left\{B_{1 \varepsilon}, B_{2 \varepsilon}\right\}
$$


where

$$
\begin{aligned}
B_{1 \varepsilon}= & \frac{r_{1}^{l}-a_{12}^{u}\left(M_{2}+\varepsilon\right)-a_{13}^{u}\left(M_{3}+\varepsilon\right)}{a_{11}^{u} \exp \left\{-\left[r_{1}^{l}-a_{11}^{u}\left(M_{1}+\varepsilon\right)-a_{12}^{u}\left(M_{2}+\varepsilon\right)-a_{13}^{u}\left(M_{3}+\varepsilon\right)\right] \tau^{u}\right\}} \\
& \times \exp \left\{r_{1}^{l}-a_{12}^{u}\left(M_{2}+\varepsilon\right)-a_{13}^{u}\left(M_{3}+\varepsilon\right)\right. \\
& \left.-a_{11}^{u} \exp \left\{-\left[r_{1}^{l}-a_{11}^{u}\left(M_{1}+\varepsilon\right)-a_{12}^{u}\left(M_{2}+\varepsilon\right)-a_{13}^{u}\left(M_{3}+\varepsilon\right)\right] \tau^{u}\right\}\right\} M_{1}, \\
B_{2 \varepsilon}= & \frac{r_{2}^{u}-a_{23}^{u}\left(M_{3}+\varepsilon\right)}{a_{22}^{u} \exp \left\{\left[r_{2}^{u}-a_{21}^{l}\left(m_{1}-\varepsilon\right)+a_{22}^{u}\left(M_{2}+\varepsilon\right)+a_{23}^{u}\left(M_{3}+\varepsilon\right)\right] \tau^{u}\right\}} .
\end{aligned}
$$

Setting $\varepsilon \rightarrow 0$ in the above inequality, we have that

$$
\lim _{k \rightarrow+\infty} \inf x_{2}(k) \geq \frac{1}{2} \min \left\{B_{1}, B_{2}\right\}:=m_{2}
$$

where

$$
\begin{aligned}
B_{1}= & \frac{r_{1}^{l}-a_{12}^{u} M_{2}-a_{13}^{u} M_{3}}{a_{11}^{u} \exp \left\{-\left[r_{1}^{l}-a_{11}^{u} M_{1}-a_{12}^{u} M_{2}-a_{13}^{u} M_{3}\right] \tau^{u}\right\}} \\
& \times \exp \left\{r_{1}^{l}-a_{12}^{u} M_{2}-a_{13}^{u} M_{3}\right. \\
& \left.-a_{11}^{u} \exp \left\{-\left[r_{1}^{l}-a_{11}^{u} M_{1}-a_{12}^{u} M_{2}-a_{13}^{u} M_{3}\right] \tau^{u}\right\}\right\} M_{1}, \\
B_{2}= & \frac{r_{2}^{u}-a_{23}^{u} M_{3}}{a_{22}^{u} \exp \left\{\left[r_{2}^{u}-a_{21}^{l} m_{1}+a_{22}^{u} M_{2}+a_{23}^{u} M_{3}\right] \tau^{u}\right\}} .
\end{aligned}
$$

For $k \geq N_{1}+\tau^{u}$, it follows from the third equation of system (1.4) that

$$
\begin{aligned}
x_{3}(k+1)= & x_{3}(k)\left[-r_{3}(k)+a_{31}(k) x_{1}\left(k-\tau_{31}(k)\right)\right. \\
& \left.-a_{32}(k) x_{2}\left(k-\tau_{32}(k)\right)-a_{33}(k) x_{3}\left(k-\tau_{33}(k)\right)\right] \\
\geq & x_{3}(t)\left[-r_{3}^{u}+a_{31}^{l}\left(m_{1}-\varepsilon\right)-a_{32}^{u}\left(M_{2}+\varepsilon\right)-a_{33}^{u}\left(M_{3}+\varepsilon\right)\right] .
\end{aligned}
$$

Hence

$$
x_{3}\left(k-\tau_{33}(k)\right) \leq x_{3}(k) \exp \left\{\left[r_{3}^{u}-a_{31}^{l}\left(m_{1}-\varepsilon\right)+a_{32}^{u}\left(M_{2}+\varepsilon\right)+a_{33}^{u}\left(M_{3}+\varepsilon\right)\right] \tau^{u}\right\} .
$$

Substituting (2.27) into the third equation of system (1.4), we derive

$$
\begin{aligned}
x_{3}(k+1) \geq & x_{3}(k)\left\{-r_{3}^{u}+a_{31}^{l}\left(m_{1}-\varepsilon\right)-a_{32}\left(M_{2}+\varepsilon\right)\right. \\
& \left.-a_{33} \exp \left\{\left[r_{3}^{u}-a_{31}^{l}\left(m_{1}-\varepsilon\right)+a_{32}^{u}\left(M_{2}+\varepsilon\right)+a_{33}^{u}\left(M_{3}+\varepsilon\right)\right] \tau^{u}\right\} x_{3}(k)\right\} .
\end{aligned}
$$

In view of Lemma 2.2 and (2.28), one has

$$
\lim _{K \rightarrow+\infty} \inf x_{3}(k) \geq \min \left\{C_{1 \varepsilon}, C_{2 \varepsilon}\right\}
$$


where

$$
\begin{aligned}
C_{1 \varepsilon}= & \frac{r_{1}^{l}-a_{12}^{u}\left(M_{2}+\varepsilon\right)-a_{13}^{u}\left(M_{3}+\varepsilon\right)}{a_{11}^{u} \exp \left\{-\left[r_{1}^{l}-a_{11}^{u}\left(M_{1}+\varepsilon\right)-a_{12}^{u}\left(M_{2}+\varepsilon\right)-a_{13}^{u}\left(M_{3}+\varepsilon\right)\right] \tau^{u}\right\}} \\
& \times \exp \left\{-r_{3}^{u}+a_{31}^{l}\left(m_{1}-\varepsilon\right)-a_{32}^{u}\left(M_{2}+\varepsilon\right)\right. \\
& \left.-a_{33} \exp \left\{\left[r_{3}^{u}-a_{31}^{l}\left(m_{1}-\varepsilon\right)+a_{32}^{u}\left(M_{2}+\varepsilon\right)+a_{33}^{u}\left(M_{3}+\varepsilon\right)\right] \tau^{u}\right\}\right\} M_{1}, \\
C_{2 \varepsilon}= & \frac{-r_{3}^{u}+a_{31}^{l}\left(m_{1}-\varepsilon\right)-a_{32}^{u}\left(M_{2}+\varepsilon\right)}{a_{33} \exp \left\{\left[r_{3}^{u}-a_{31}^{l}\left(m_{1}-\varepsilon\right)+a_{32}^{u}\left(M_{2}+\varepsilon\right)+a_{33}^{u}\left(M_{3}+\varepsilon\right)\right] \tau^{u}\right\}} .
\end{aligned}
$$

Setting $\varepsilon \rightarrow 0$ in (2.29) leads to

$$
\lim _{k \rightarrow+\infty} \inf x_{3}(k) \geq \frac{1}{2} \min \left\{C_{1}, C_{2}\right\}:=m_{3},
$$

where

$$
\begin{aligned}
C_{1}= & \frac{r_{1}^{l}-a_{12}^{u} M_{2}-a_{13}^{u} M_{3}}{a_{11}^{u} \exp \left\{-\left[r_{1}^{l}-a_{11}^{u} M_{1}-a_{12}^{u} M_{2}-a_{13}^{u} M_{3}\right] \tau^{u}\right\}} \\
& \times \exp \left\{-r_{3}^{u}+a_{31}^{l} m_{1}-a_{32}^{u} M_{2}\right. \\
& \left.-a_{33} \exp \left\{\left[r_{3}^{u}-a_{31}^{l} m_{1}+a_{32}^{u} M_{2}+a_{33}^{u} M_{3}\right] \tau^{u}\right\}\right\} M_{1}, \\
C_{2}= & \frac{-r_{3}^{u}+a_{31}^{l} m_{1}-a_{32}^{u} M_{2}}{a_{33} \exp \left\{\left[r_{3}^{u}-a_{31}^{l} m_{1}+a_{32}^{u} M_{2}+a_{33}^{u} M_{3}\right] \tau^{u}\right\}} .
\end{aligned}
$$

In view of (2.4), (2.10), (2.15), (2.20), (2.25) and (2.30), we can conclude that system (1.4) is permanent. The proof of Theorem 2.1 is complete.

Remark 2.1 Under the assumption of Theorem 2.1, the set $\left[m_{1}, M_{1}\right] \times\left[m_{2}, M_{2}\right] \times\left[m_{3}, M_{3}\right]$ is an invariant set of system (1.4).

\section{Global stability}

In this section, we formulate the stability property of positive solutions of system (1.4) when all the time delays are zero.

Theorem 3.1 Let $\tau_{i j}=0(i, j=1,2,3)$. In addition to $(\mathrm{H} 1)-(\mathrm{H} 3)$, assume further that $(\mathrm{H} 4)$

$$
\begin{aligned}
& \delta_{1}=\max \left\{\left|1-a_{11}^{u} M_{1}\right|,\left|1-a_{11}^{l} m_{1}\right|\right\}+a_{12}^{u} M_{2}+a_{13}^{u} M_{3}<1, \\
& \delta_{2}=\max \left\{\left|1-a_{22}^{u} M_{2}\right|,\left|1-a_{22}^{l} m_{3}\right|\right\}+a_{21}^{u} M_{1}+a_{23}^{u} M_{3}<1, \\
& \delta_{3}=\max \left\{\left|1-a_{33}^{u} M_{3}\right|,\left|1-a_{33}^{l} m_{3}\right|\right\}+a_{31}^{u} M_{1}+a_{32}^{u} M_{2}<1 .
\end{aligned}
$$

Then, for any positive solution $\left(x_{1}(k), x_{2}(k), x_{3}(k)\right)$ and $\left(x_{1}^{*}(k), x_{2}^{*}(k), x_{3}^{*}(k)\right)$ of system (1.4), we have

$$
\lim _{k \rightarrow \infty}\left[x_{i}^{*}(k)-x_{i}(k)\right]=0 \quad(i=1,2,3) .
$$


Proof Let

$$
x_{i}(k)=x_{i}^{*}(k) \exp \left(u_{i}(k)\right), \quad i=1,2,3 .
$$

Then system (1.4) is equivalent to

$$
\left\{\begin{aligned}
u_{1}(k+1)= & u_{1}(k)-a_{11}(k) x_{1}^{*}(k)\left(\exp \left(u_{1}(k)\right)-1\right) \\
& -a_{12}(k) x_{2}^{*}(k)\left(\exp \left(u_{2}(k)\right)-1\right) \\
& -a_{13}(k) x_{3}^{*}(k)\left(\exp \left(u_{3}(k)\right)-1\right) \\
u_{2}(k+1)= & u_{2}(k)+a_{21}(k) x_{1}^{*}(k)\left(\exp \left(u_{1}(k)\right)-1\right) \\
& -a_{22}(k) x_{2}^{*}(k)\left(\exp \left(u_{2}(k)\right)-1\right) \\
& -a_{23}(k) x_{3}^{*}(k)\left(\exp \left(u_{3}(k)\right)-1\right) \\
u_{3}(k+1)= & u_{3}(k)-a_{31}(k) x_{1}^{*}(k)\left(\exp \left(u_{1}(k)\right)-1\right) \\
& -a_{32}(k) x_{2}^{*}(k)\left(\exp \left(u_{2}(k)\right)-1\right) \\
& -a_{33}(k) x_{3}^{*}(k)\left(\exp \left(u_{3}(k)\right)-1\right)
\end{aligned}\right.
$$

Then

$$
\left\{\begin{aligned}
u_{1}(k+1)= & u_{1}(k)-a_{11}(k) x_{1}^{*}(k)\left(\exp \left(\theta_{11}(k) u_{1}(k)\right) u_{1}(k)\right. \\
& -a_{12}(k) x_{2}^{*}(k)\left(\exp \left(\theta_{12}(k) u_{2}(k)\right) u_{2}(k)\right. \\
& -a_{13}(k) x_{3}^{*}(k)\left(\exp \left(\theta_{13}(k) u_{3}(k)\right) u_{3}(k),\right. \\
u_{2}(k+1)= & u_{2}(k)+a_{21}(k) x_{1}^{*}(k)\left(\exp \left(\theta_{21}(k) u_{1}(k)\right) u_{1}(k)\right. \\
& -a_{22}(k) x_{2}^{*}(k)\left(\exp \left(\theta_{22}(k) u_{2}(k)\right) u_{2}(k)\right. \\
& -a_{23}(k) x_{3}^{*}(k)\left(\exp \left(\theta_{23}(k) u_{3}(k)\right) u_{3}(k),\right. \\
u_{3}(k+1)= & u_{3}(k)+a_{31}(k) x_{1}^{*}(k)\left(\exp \left(\theta_{31}(k) u_{1}(k)\right) u_{1}(k)\right. \\
& -a_{32}(k) x_{2}^{*}(k)\left(\exp \left(\theta_{32}(k) u_{2}(k)\right) u_{2}(k)\right. \\
& -a_{33}(k) x_{3}^{*}(k)\left(\exp \left(\theta_{33}(k) u_{3}(k)\right) u_{3}(k),\right.
\end{aligned}\right.
$$

where $\theta_{i j}(k) \in[0,1](i, j=1,2,3)$. To complete the proof, it suffices to show that

$$
\lim _{k \rightarrow+\infty} u_{i}(k)=0 \quad(i=1,2,3)
$$

In view of (H4), we can choose $\varepsilon>0$ small enough such that

$$
\begin{aligned}
& \delta_{1}^{\varepsilon}=\max \left\{\left|1-a_{11}^{u}\left(M_{1}+\varepsilon\right)\right|,\left|1-a_{11}^{l}\left(m_{1}-\varepsilon\right)\right|\right\}+a_{12}^{u}\left(M_{2}+\varepsilon\right)+a_{13}^{u}\left(M_{3}+\varepsilon\right)<1, \\
& \delta_{2}^{\varepsilon}=\max \left\{\left|1-a_{22}^{u}\left(M_{2}+\varepsilon\right)\right|,\left|1-a_{22}^{l}\left(m_{3}-\varepsilon\right)\right|\right\}+a_{21}^{u}\left(M_{1}+\varepsilon\right)+a_{23}^{u}\left(M_{3}+\varepsilon\right)<1, \\
& \delta_{3}^{\varepsilon}=\max \left\{\left|1-a_{33}^{u}\left(M_{3}+\varepsilon\right)\right|,\left|1-a_{33}^{l}\left(m_{3}-\varepsilon\right)\right|\right\}+a_{31}^{u}\left(M_{1}+\varepsilon\right)+a_{32}^{u}\left(M_{2}+\varepsilon\right)<1 .
\end{aligned}
$$

For above $\varepsilon>0$, in view of Theorem 2.1 in Section 2, there exists $k^{*} \in N$ such that

$$
m_{i}-\varepsilon \leq x_{i}^{*}(k), x_{i}(k) \leq M_{i}+\varepsilon \quad(i=1,2,3)
$$

for all $k \geq k^{*}$. 
Noticing that $\theta_{i j}(k) \in[0,1](i, j=1,2,3)$ implies that $x_{i}^{*}(k) \exp \left(\theta_{j i}(k) u_{i}(k)\right)$ lies between $x_{i}^{*}(k)$ and $x_{i}(k)$. From (3.3), we have

$$
\begin{aligned}
u_{1}(k+1) \leq & \max \left\{\left|1-a_{11}^{u}\left(M_{1}+\varepsilon\right)\right|,\left|1-a_{11}^{l}\left(m_{1}-\varepsilon\right)\right|\right\}\left|u_{1}(k)\right| \\
& +a_{12}^{u}\left(M_{2}+\varepsilon\right)\left|u_{2}(k)\right|+a_{13}^{u}\left(M_{3}+\varepsilon\right)\left|u_{3}(k)\right|, \\
u_{2}(k+1) \leq & \max \left\{\left|1-a_{22}^{u}\left(M_{2}+\varepsilon\right)\right|,\left|1-a_{22}^{l}\left(m_{3}-\varepsilon\right)\right|\right\}\left|u_{2}(k)\right| \\
& +a_{21}^{u}\left(M_{1}+\varepsilon\right)\left|u_{1}(k)\right|+a_{23}^{u}\left(M_{3}+\varepsilon\right)\left|u_{3}(k)\right|, \\
u_{3}(k+1) \leq & \max \left\{\left|1-a_{33}^{u}\left(M_{3}+\varepsilon\right)\right|,\left|1-a_{33}^{l}\left(m_{3}-\varepsilon\right)\right|\right\}\left|u_{3}(k)\right| \\
& +a_{31}^{u}\left(M_{1}+\varepsilon\right)\left|u_{1}(k)\right|+a_{32}^{u}\left(M_{2}+\varepsilon\right)\left|u_{2}(k)\right| .
\end{aligned}
$$

Let $\rho=\max \left\{\delta_{1}^{\varepsilon}, \delta_{2}^{\varepsilon}, \delta_{3}^{\varepsilon}\right\}$, then $\rho<1$. By (3.8)-(3.10), for $k \geq k^{*}$, we have

$$
\max \left\{\left|u_{1}(k+1)\right|,\left|u_{2}(k+1)\right|,\left|u_{3}(k+1)\right|\right\} \leq \rho \max \left\{\left|u_{1}(k)\right|,\left|u_{2}(k)\right|,\left|u_{3}(k)\right|\right\},
$$

which implies

$$
\max \left\{\left|u_{1}(k)\right|,\left|u_{2}(k)\right|,\left|u_{3}(k)\right|\right\} \leq \rho^{k-k^{*}} \max \left\{\left|u_{1}\left(k^{*}\right)\right|,\left|u_{2}\left(k^{*}\right)\right|,\left|u_{3}\left(k^{*}\right)\right|\right\} .
$$

Thus (3.4) holds true and the proof is complete.

\section{Existence and stability of a periodic solution}

In this section, we further assume that $\tau_{i j}=0(i, j=1,2,3)$ and the coefficients of system (1.4) satisfy the following condition:

(H5) There exists a positive integer $\omega$ such that for $k \in N, 0<r_{i}(k+\omega)=r_{i}(k)$,

$$
0<a_{i j}(k+\omega)=a_{i j}(k)(i, j=1,2,3) .
$$

Theorem 4.1 Assume that (H1)-(H5) are satisfied, then system (1.4) with all the delays $\tau_{i j}=0(i, j=1,2,3)$ admits at least one positive $\omega$-periodic solution which we denote by $\left(x_{1}^{*}(k), x_{2}^{*}(k), x_{3}^{*}(k)\right)$.

Proof As pointed out in Remark 2.1 of Section 2,

$$
D^{3} \stackrel{\text { def }}{=}\left[m_{1}, M_{1}\right] \times\left[m_{2}, M_{2}\right] \times\left[m_{3}, M_{3}\right]
$$

is an invariant set of system (1.4). Then we define a mapping $F$ on $D^{3}$ by

$$
F\left(x_{1}(0), x_{2}(0), x_{3}(0)\right)=\left(x_{1}(\omega), x_{2}(\omega), x_{3}(\omega)\right), \quad \text { for }\left(x_{1}(0), x_{2}(0), x_{3}(0)\right) \in D^{3} .
$$

Clearly, $F$ depends continuously on $\left(x_{1}(0), x_{2}(0), x_{3}(0)\right)$. Thus $F$ is continuous and maps the compact set $D^{3}$ into itself. Therefore, $F$ has a fixed point. It is not difficult to see that the solution $\left(x_{1}^{*}(k), x_{2}^{*}(k), x_{3}^{*}(k)\right)$ passing through this fixed point is an $\omega$-periodic solution of system (1.4). The proof of Theorem 4.1 is complete.

Theorem 4.2 Assume that (H1)-(H5) are satisfied, then system (1.4) with all the delays $\tau_{i j}=0(i, j=1,2,3)$ has a globally stable positive $\omega$-periodic solution. 
Proof Under assumptions (H1)-(H5), it follows from Theorem 4.1 that system (1.4) with all the delays $\tau_{i j}=0(i, j=1,2,3)$ admits at least one positive $\omega$-periodic solution. In addition, Theorem 3.1 ensures that the positive solution is globally stable. Hence the proof.

\section{Numerical example}

In this section, we will give an example which shows the feasibility of the main results (Theorem 2.1) of this paper. Let us consider the following discrete system:

$$
\left\{\begin{aligned}
x_{1}(k+1)= & x_{1}(k) \exp \left\{\left(0.5+0.03 \sin \frac{k \pi}{2}\right)-\left(0.4+0.02 \sin \frac{k \pi}{2}\right) x_{1}(k-1)\right. \\
& \left.-\left(0.04+0.02 \cos \frac{k \pi}{2}\right) x_{2}(k-1)-\left(0.03+0.02 \cos \frac{k \pi}{2}\right) x_{3}(k-1)\right\} \\
x_{2}(k+1)= & x_{2}(k) \exp \left\{-\left(0.085+0.05 \cos \frac{k \pi}{2}\right)+\left(0.5+0.3 \sin \frac{k \pi}{2}\right) x_{1}(k-1)\right. \\
& \left.-\left(0.26+0.02 \sin \frac{k \pi}{2}\right) x_{2}(k-1)-\left(0.5+0.04 \cos \frac{k \pi}{2}\right) x_{3}(k-1)\right\} \\
x_{3}(k+1)= & x_{3}(k) \exp \left\{-\left(0.075+0.03 \sin \frac{k \pi}{2}\right)+\left(0.5+0.02 \cos \frac{k \pi}{2}\right) x_{1}(k-1)\right. \\
& \left.-\left(0.24+0.04 \sin \frac{k \pi}{2}\right) x_{2}(k-1)-\left(0.4+0.06 \cos \frac{k \pi}{2}\right) x_{3}(k-1)\right\}
\end{aligned}\right.
$$

Here

$$
\begin{aligned}
& r_{1}(k)=0.5+0.03 \sin \frac{k \pi}{2}, \quad r_{2}(t)=0.085+0.05 \cos \frac{k \pi}{2}, \\
& r_{3}(t)=0.075+0.03 \sin \frac{k \pi}{2}, \\
& a_{11}(k)=0.4+0.02 \sin \frac{k \pi}{2}, \quad a_{12}(k)=0.03+0.02 \cos \frac{k \pi}{2}, \\
& a_{13}(k)=0.03+0.02 \cos \frac{k \pi}{2}, \\
& a_{21}(k)=0.5+0.3 \sin \frac{k \pi}{2}, \quad a_{22}(k)=0.26+0.02 \sin \frac{k \pi}{2}, \\
& a_{23}(k)=0.5+0.04 \cos \frac{k \pi}{2}, \\
& a_{31}(k)=0.5+0.02 \cos \frac{k \pi}{2}, \\
& a_{33}(k)=0.4+0.06 \cos \frac{k \pi}{2}, \\
& \tau_{i j}(k)=1 \quad(i, j=1,2,3) .
\end{aligned}
$$

All the coefficients $r_{i}(k)(i=1,2,3), a_{i j}(k)(i, j=1,2,3), \tau_{i j}(k)(i, j=1,2,3)$ are functions with respect to $k$, and it is not difficult to obtain that

$$
\left\{\begin{array}{l}
r_{1}^{u}=0.53, \quad r_{1}^{l}=0.47, \quad r_{2}^{u}=0.135, \quad r_{2}^{l}=0.035, \\
r_{3}^{u}=0.105, \quad r_{3}^{l}=0.045, \\
a_{11}^{u}=0.402, \quad a_{11}^{l}=0.038, \quad a_{12}^{u}=0.05, \quad a_{12}^{l}=0.01, \\
a_{13}^{u}=0.05, \quad a_{13}^{l}=0.01, \\
a_{21}^{u}=0.8, \quad a_{21}^{l}=0.3, \quad a_{22}^{u}=0.28, \quad a_{22}^{l}=0.24, \\
a_{23}^{u}=0.54, \quad a_{23}^{l}=0.46, \\
a_{31}^{u}=0.52, \quad a_{31}^{l}=0.48, \quad a_{32}^{u}=0.28, \quad a_{32}^{l}=0.20, \\
a_{33}^{u}=0.46, \quad a_{34}^{l}=0.34, \\
\tau_{i j}^{u}=\tau_{i j}^{l}=1 \quad(i, j=1,2,3) .
\end{array}\right.
$$




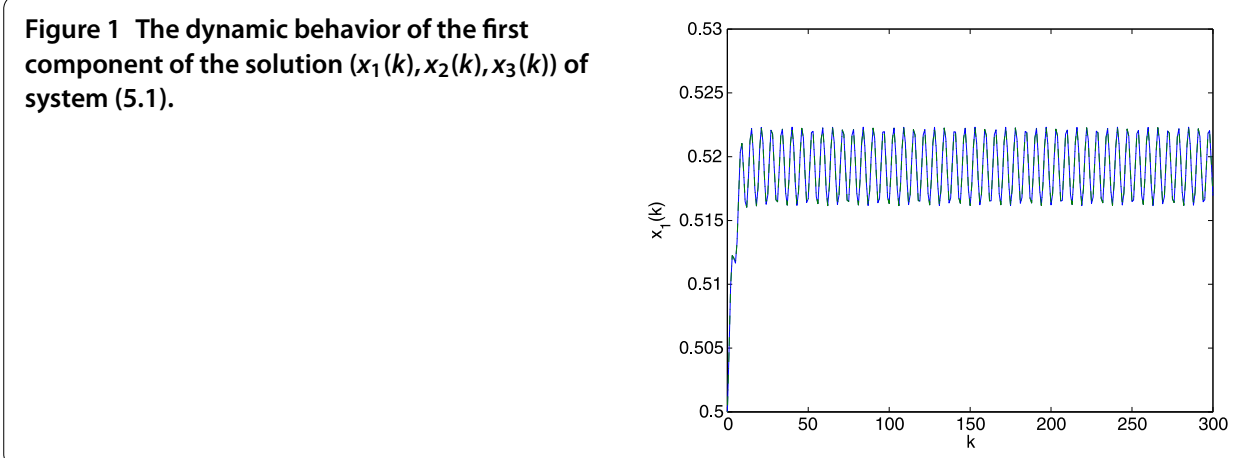

Figure 2 The dynamic behavior of the second component of the solution $\left(x_{1}(k), x_{2}(k), x_{3}(k)\right)$ of system (5.1).

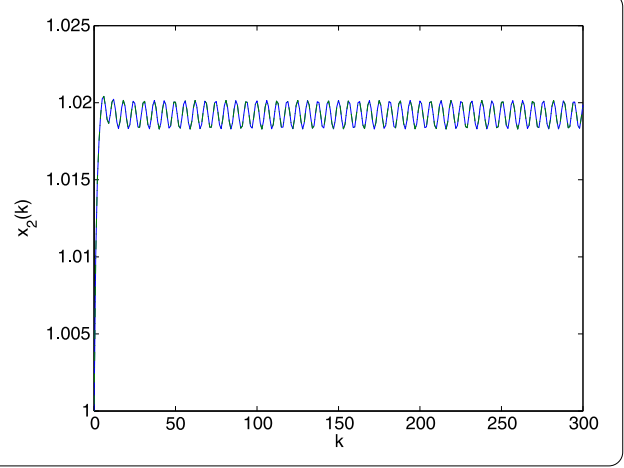

Figure 3 The dynamic behavior of the third component of the solution $\left(x_{1}(k), x_{2}(k), x_{3}(k)\right)$ of system (5.1).

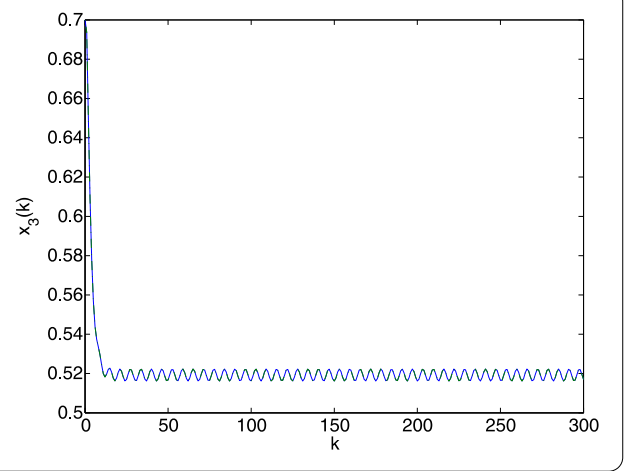

It is easy to examine that the coefficients of system (5.1) satisfy all the conditions of Theorem 2.1. Thus system (5.1) is permanent which is shown in Figures 1-3.

\section{Conclusions}

In this paper, we have investigated the dynamic behavior of a discrete Lotka-Volterra predator-prey model with time-varying delays. Sufficient conditions which ensure the permanence of the system are established. Moreover, we also analyze the global stability of the system with all the delays $\tau_{i j}(k)=0(i, j=1,2,3)$ and deal with the existence and stability of the system. We have shown that delay has important influence on the permanence of the system. Therefore, delay is an important factor to decide the permanence of the system. When all the delays are zero, we obtain some sufficient conditions which guarantee the global stability of the system. Computer simulations are carried out to explain our main theoretical results. 


\section{Competing interests}

The authors declare that they have no competing interests.

\section{Authors' contributions}

The authors have made the same contribution. All authors read and approved the final manuscript.

\section{Author details}

${ }^{1}$ Guizhou Key Laboratory of Economics System Simulation, School of Mathematics and Statistics, Guizhou University of Finance and Economics, Guiyang, 550004, P.R. China. ${ }^{2}$ School of Mathematics and Statistics, Henan University of Science and Technology, Luoyang, 471023, P.R. China.

\section{Acknowledgements}

The first author was supported by the National Natural Science Foundation of China (No. 11261010), the Soft Science and Technology Program of Guizhou Province (No. 2011LKC2030), the Natural Science and Technology Foundation of Guizhou Province (J[2012]2100), the Governor Foundation of Guizhou Province ([2012]53) and the Doctoral Foundation of Guizhou University of Finance and Economics (2010). The second author was supported by the National Natural Science Foundation of China (No. 11101126). The third author was supported by the Natural Science Innovation Team Project of Guizhou Province ([2013]14). The authors would like to thank the referees and the editor for helpful suggestions incorporated into this paper.

\section{Received: 12 February 2014 Accepted: 7 July 2014 Published: 04 Aug 2014}

\section{References}

1. Berryman, AA: The origins and evolution of predator-prey theory. Ecology 73(5), 1530-1535 (1992)

2. Dai, BX, Zou, JZ: Periodic solutions of a discrete-time diffusive system governed by backward difference equations. Adv. Differ. Equ. 2005, Article ID 586218 (2005)

3. Gyllenberg, M, Yan, P, Wang, Y: Limit cycles for competitor-competitor-mutualist Lotka-Volterra systems. Physica D 221(2), 135-145 (2006)

4. Fan, M, Wang, K: Periodic solutions of a discrete time nonautonomous ratio-dependent predator-prey system. Math. Comput. Model. 35(9-10), 951-961 (2002)

5. Fazly, M, Hesaaraki, M: Periodic solutions for a discrete time predator-prey system with monotone functional responses. C. R. Math. Acad. Sci. Paris 345(4), 199-202 (2007)

6. Gaines, RE, Mawhin, JL: Coincidence Degree and Nonlinear Differential Equations. Springer, Berlin (1997)

7. Kar, TK, Ghorai, A: Dynamic behaviour of a delayed predator-prey model with harvesting. Appl. Math. Comput. 217(22), 9085-9104 (2011)

8. Sen, M, Banerjee, M, Morozov, A: Bifurcation analysis of a ratio-dependent prey-predator model with the Allee effect. Ecol. Complex. 11, 12-27 (2012)

9. Haque, M, Venturino, E: An ecoepidemiological model with disease in predator: the ratio-dependent case. Math. Methods Appl. Sci. 30(14), 1791-1809 (2007)

10. Braza, PA: Predator-prey dynamics with square root functional responses. Nonlinear Anal., Real World Appl. 13(4), 1837-1843 (2012)

11. Wiener, J: Differential equations with piecewise constant delays. In: Trends in Theory and Practice of Nonlinear Differential Equations. Lecture Notes in Pure and Applied Mathematics, vol. 90. Dekker, New York (1984)

12. Xu, R, Chen, LS, Hao, FL: Periodic solution of a discrete time Lotka-Volterra type food-chain model with delays. Appl. Math. Comput. 171(1), 91-103 (2005)

13. Zhang, JB, Fang, H: Multiple periodic solutions for a discrete time model of plankton allelopathy. Adv. Differ. Equ. 2006, Article ID 90479 (2006)

14. Xiong, XS, Zhang, ZQ: Periodic solutions of a discrete two-species competitive model with stage structure. Math. Comput. Model. 48(3-4), 333-343 (2008)

15. Zhang, RY, Wang, ZC, Chen, YM, Wu, JH: Periodic solutions of a single species discrete population model with periodic harvest/stock. Comput. Math. Appl. 39(1-2), 77-90 (2009)

16. Zhang, WP, Zhu, DM, Bi, P: Multiple periodic positive solutions of a delayed discrete predator-prey system with type IV functional responses. Appl. Math. Lett. 20(10), 1031-1038 (2007)

17. Zhang, ZQ, Luo, JB: Multiple periodic solutions of a delayed predator-prey system with stage structure for the predator. Nonlinear Anal., Real World Appl. 11(5), 4109-4120 (2010)

18. Li, YK, Zhao, KH, Ye, Y: Multiple positive periodic solutions of species delay competition systems with harvesting terms. Nonlinear Anal., Real World Appl. 12(2), 1013-1022 (2011)

19. Sun, YG, Saker, SH: Positive periodic solutions of discrete three-level food-chain model of Holling type II. Appl. Math. Comput. 180(1), 353-365 (2006)

20. Ding, $\mathrm{XH}$, Liu, C: Existence of positive periodic solution for ratio-dependent $\mathrm{N}$-species difference system. Appl. Math. Model. 33(6), 2748-2756 (2009)

21. Chakraborty, K, Chakraborty, M, Kar, TK: Bifurcation and control of a bioeconomic model of a prey-predator system with a time delay. Nonlinear Anal. Hybrid Syst. 5(4), 613-625 (2011)

22. Li, ZC, Zhao, QL, Ling, D: Chaos in a discrete population model. Discrete Dyn. Nat. Soc. 2012, Article ID 482459 (2012)

23. Xiang, H, Yang, KM, Wang, BY: Existence and global stability of periodic solution for delayed discrete high-order Hopfield-type neural networks. Discrete Dyn. Nat. Soc. 2005(3), 281-297 (2005)

24. Gopalsamy, K: Stability and Oscillations in Delay Differential Equations of Population Dynamics. Kluwer Academic, Dordrecht (1992)

25. Kuang, Y: Delay Differential Equations with Applications in Population Dynamics. Academic Press, New York (1993)

26. Fan, L, Shi, ZK, Tang, SY: Critical values of stability and Hopf bifurcations for a delayed population model with delay-dependent parameters. Nonlinear Anal., Real World Appl. 11(1), 341-355 (2010)

27. Fan, YH, Li, WT: Permanence for a delayed discrete ratio-dependent predator-prey model with Holling type functional response. J. Math. Anal. Appl. 299(2), 357-374 (2004) 
28. Chen, FD: Permanence of a discrete $n$-species food-chain system with time delays. Appl. Math. Comput. 185(1), 719-726 (2007)

29. Zhao, JD, Jiang, JF: Average conditions for permanence and extinction in nonautonomous Lotka-Volterra system. J. Math. Anal. Appl. 299(2), 663-675 (2004)

30. Chen, FD: The permanence and global attractivity of Lotka-Volterra competition system with feedback control. Nonlinear Anal., Real World Appl. 7(1), 133-143 (2006)

31. Teng, ZD, Zhang, Y, Gao, SJ: Permanence criteria for general delayed discrete nonautonomous $n$-species Kolmogorov systems and its applications. Comput. Math. Appl. 59(2), 812-828 (2010)

32. Dhar, J, Jatav, KS: Mathematical analysis of a delayed stage-structured predator-prey model with impulsive diffusion between two predators territories. Ecol. Complex. 16, 59-67 (2013)

33. Liu, SQ, Chen, LS: Necessary-sufficient conditions for permanence and extinction in Lotka-Volterra system with distributed delay. Appl. Math. Lett. 16(6), 911-917 (2003)

34. Liao, XY, Zhou, SF, Chen, YM: Permanence and global stability in a discrete $n$-species competition system with feedback controls. Nonlinear Anal., Real World Appl. 9(4), 1661-1671 (2008)

35. Hu, HX, Teng, ZD, Jiang, HJ: On the permanence in non-autonomous Lotka-Volterra competitive system with pure-delays and feedback controls. Nonlinear Anal., Real World Appl. 10(3), 1803-1815 (2009)

36. Muroya, Y: Permanence and global stability in a Lotka-Volterra predator-prey system with delays. Appl. Math. Lett. 16(8), 1245-1250 (2003)

37. Kuniya, T, Nakata, Y: Permanence and extinction for a nonautonomous SEIRS epidemic model. Appl. Math. Comput. 218(18), 9321-9331 (2012)

38. Hou, ZY: On permanence of Lotka-Volterra systems with delays and variable intrinsic growth rates. Nonlinear Anal., Real World Appl. 14(2), 960-975 (2013)

39. Li, CH, Tsai, CC, Yang, SY: Analysis of the permanence of an SIR epidemic model with logistic process and distributed time delay. Commun. Nonlinear Sci. Numer. Simul. 17(9), 3696-3707 (2012)

40. Chen, FD, You, MS: Permanence for an integrodifferential model of mutualism. Appl. Math. Comput. 186(1), 30-34 (2007)

41. Berezansky, L, Baštinec, J, Diblík, J, Šmarda, Z: On a delay population model with quadratic nonlinearity. Adv. Differ Equ. 2012, Article ID 230 (2012). doi:10.1186/1687-1847-2012-230

42. Baštinec, J, Berezansky, L, Diblík, J, Šmarda, Z: On a delay population model with a quadratic nonlinearity without positive steady state. Appl. Math. Comput. 227, 622-629 (2014)

43. Tang, XH, Cao, DM, Zou, XF: Global attractivity of positive periodic solution to periodic Lotka-Volterra competition systems with pure delay. J. Differ. Equ. 228(2), 580-610 (2006)

44. Tang, XH, Zou, XF: Global attractivity in a predator-prey system with pure delays. Proc. Edinb. Math. Soc. 51, 495-508 (2008)

45. LV, X, Lu, SP, Yan, P: Existence and global attractivity of positive periodic solutions of Lotka-Volterra predator-prey systems with deviating arguments. Nonlinear Anal., Real World Appl. 11(5-6), 574-583 (2010)

46. Chen, YM, Zhou, ZF: Stable periodic of a discrete periodic Lotka-Volterra competition system. J. Math. Anal. Appl. 277(1), 358-366 (2003)

47. Chen, FD: Permanence for the discrete mutualism model with time delays. Math. Comput. Model. 47(3-4), 431-435 (2008)

10.1186/1687-1847-2014-208

Cite this article as: $\mathrm{Xu}$ et al.: Permanence and global attractivity in a discrete Lotka-Volterra predator-prey mode with delays. Advances in Difference Equations 2014, 2014:208

\section{Submit your manuscript to a SpringerOpen ${ }^{\circ}$ journal and benefit from:}

- Convenient online submission

Rigorous peer review

- Immediate publication on acceptance

- Open access: articles freely available online

- High visibility within the field

- Retaining the copyright to your article 